\title{
Thermophotovoltaic efficiency measurement: design and analysis of a novel experimental method
}

\author{
A. Cabrera, A. Ramos, I. Artacho, M.Gomez, K. Gavin, A. Martí, A. Datas
}

\begin{abstract}
Thermophotovoltaic (TPV) devices produce the direct conversion of radiant heat into electricity using infrared sensitive photovoltaic (PV) cells. Despite its relatively mature stage of development a standardized method for the measurement of the TPV conversion efficiency hasn't been established yet, which represents a serious issue for the TPV research development. In this work we present results on a novel method to directly measure the conversion efficiency of a TPV device. This method relies on the direct measurement of the electrical power and the heat dissipated by the TPV cell in the steady state. In this article, we present preliminary results on the efficiency of a TPV device illuminated by a halogen lamp at different incident irradiances and vacuum conditions.
\end{abstract}

Keywords - Thermophotovoltaics, Conversion efficiency, GaSb, Heat flux, Experimental.

\section{INTRODUCTION}

The operation principle of thermophotovoltaic (TPV) devices is identical to that of solar photovoltaics (PV). The main difference resides in the fact that, in contrast with ordinary solar PV cells that convert the incident solar radiation to electricity, TPV cells produce the direct conversion of radiant heat coming from incandescent emitters at temperatures near or beyond $\sim 1000{ }^{\circ} \mathrm{C}[1,2]$. Thus, infrared sensitive PV cells (or TPV cells) made of $\mathrm{GaSb}, \mathrm{Ge}$, or InGaAs are typically used instead of those made of silicon or GaAs-based compounds that are used in solar PV applications. TPV devices are regarded as as a highefficiency and high-temperature alternative to thermoelectric generators (TEGs) and could be employed in virtually any kind of high temperature power generation application

One of the characteristics of operation of TPV devices is the very close operation distance from the radiant source. This distance allows the use of optical elements to reflect back to the emitter part of the radiation not absorbed in the TPV cell, enabling much higher conversion efficiencies. There exist three different types of spectral control for TPV $[3,4]$ : i) selective or filtered emitter that reduces (increases) the long-wavelength (high-wavelength) emissivity [5]; ii) filters located between the emitter and the TPV cell that transmit (reflect) the short-wavelength (long-wavelength) photons [6]; and iii) back side reflectors (BSR) located in the rear side of the TPV cell to reflect back to the emitter those photons not absorbed in the TPV cell. All these strategies aim at tuning the net radiative spectrum to match the TPV cell spectral response and consequently reduce the amount of heat that must be dissipated from the TPV cell.

One of the best performing TPV systems reported to date combines the strategies (ii) and (iii) to achieve a heat conversion efficiency of $23.6 \%$ and a power density of 0.79
$\mathrm{W} / \mathrm{cm}^{2}$, for a grey body $\mathrm{SiC}$ emitter at $1058{ }^{\circ} \mathrm{C}$ [7]. To our knowledge, the method described in $[3,7]$ is the only experimental approach reported so far for performing an accurate direct measurement of the conversion efficiency of a TPV device. Apart of this work, at the moment there is not a direct and reliable method to measure TPV efficiency. Most of the reported results estimate the product of the partial efficiencies, which include thermal radiative efficiency and PV conversion efficiency $[8,9]$. This lack of a standard and precise method to characterize TPV cells is highly detrimental for the development of TPV community, as it greatly affects the intercommunication between fellow researchers.

There might be several reasons to the absence of a standard efficiency measurement method for TPV. One of them is the great variety of applications and working conditions in which the TPV devices are employed (solar, combustion, nuclear, etc.). Each one requires the consideration and analysis of different variables as well as of other partials efficiencies particulars to each configuration. However, the main reason is probably the complex nature of the radiative exchange existing in a TPV device. The very close operation distance from the radiant source and the use of spectral control elements makes that part of the radiation impinging the TPV cell is reflected and reabsorbed into the emitter, and it shouldn't be considered as energy losses. Thus, in order to measure accurately the efficiency of a TPV device. one has to consider the net radiative flux between the emitter and the TPV cell, which form a photonic cavity, instead of just considering the incident radiation, as in the case of solar PV cells. This typically forces to use complex photonic ray tracing models [10] and applicate a cavity correction factor to determine the absorbed heat flux as a function of the radiated energy flux [9].

In this work we present preliminary results on an alternative method that allows to directly measure the conversion efficiency of a TPV device. Results of the characterization of TPV cells under halogen-lamp illumination are reported, in order to illustrate the potential of this method, which could be eventually used to measure actual TPV devices with incandescent radiant sources.

\section{EXPERIMENTAL METHOD}

The proposed method (Fig. 1) relies on the assumption that an energy balance exists in the TPV cell according to which the net radiative power on the TPV cell surface $\left(P_{\mathrm{in}}-\right.$ $\left.P_{\text {out }}\right)$ is converted to either electrical power $\left(P_{\mathrm{el}}\right)$ or dissipated heat $(Q)$ through the TPV cell. Thus, the TPV conversion efficiency can be expressed as 


$$
\eta=\frac{P_{e l}}{P_{\text {in }}-P_{\text {out }}}=\frac{P_{e l}}{P_{e l}-Q}
$$

Therefore, the TPV conversion efficiency can be calculated through the direct measurement of the heat flux from the TPV cell $(Q)$ and the output electrical power $\left(P_{\mathrm{el}}\right)$. The latter is easily measured; thus, the main difficulty of this method lays in the accurate control and measurement of the heat flux through the TPV device.

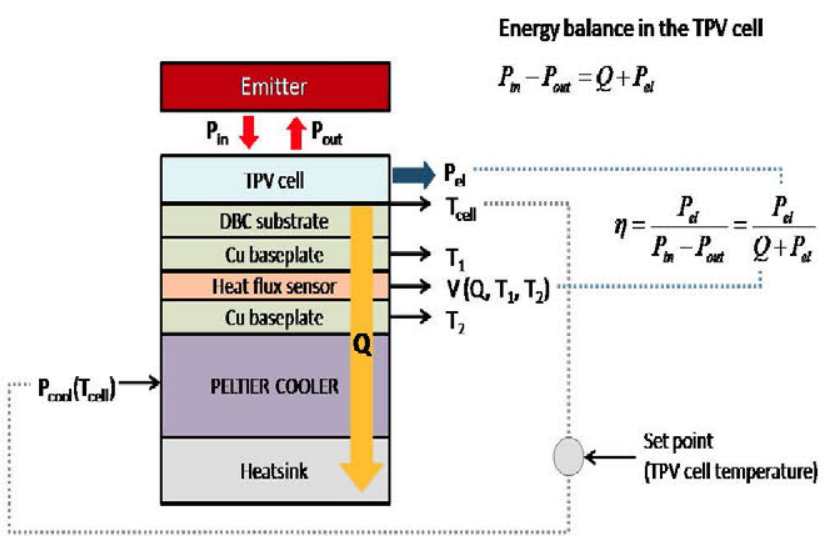

(a)

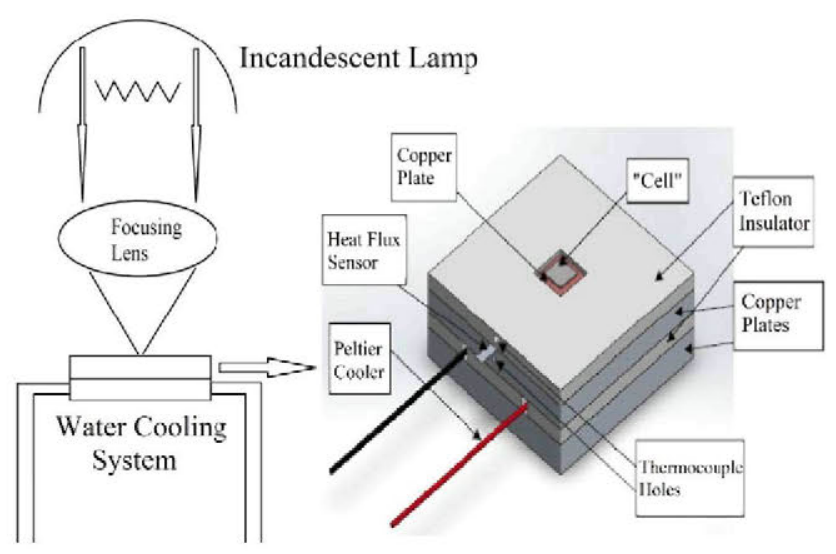

(b)

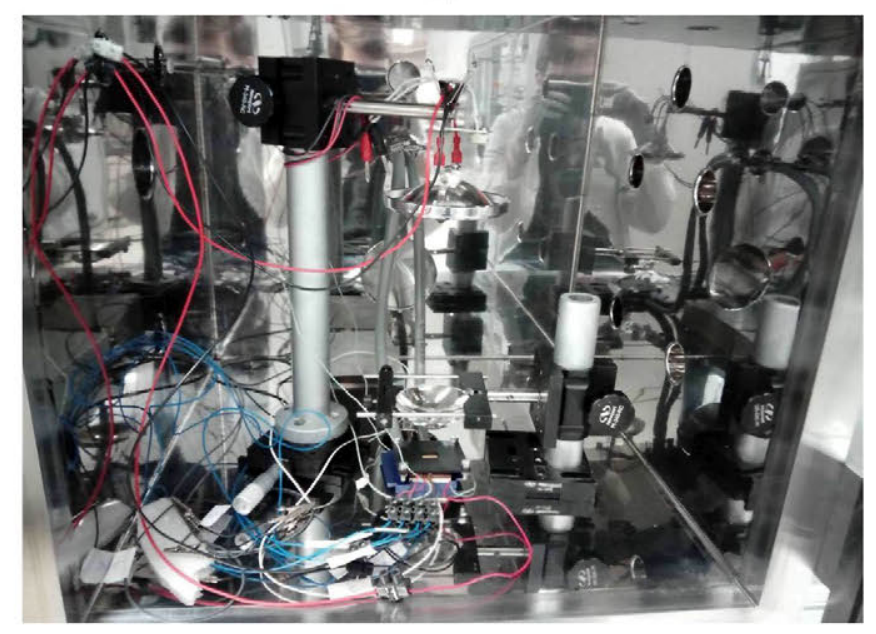

(c)

Fig. 1. The characterization setup for the measurement of the TPV conversion efficiency: (a) schematic of the TPV conversion efficiency characterization setup, (b) experimenal TPV conversion efficiency characterization setup, and (c) picture of the characterization setup inside a vacuum chamber.
In the experimental setup developed in this work, a high precision $\left(22 \mu \mathrm{V} /\left(\mathrm{W} / \mathrm{m}^{2}\right)\right)$ gSKIN $(\mathrm{B}$-XI heat flux sensor of $18.0 \times 18.0 \times 0.5 \mathrm{~mm}$ based on the Seebeck effect, is used for the heat measurement. As it can be seen in Fig. 1(a), the heat flux sensor is placed between two copper baseplate which serve to homogenize the heat flux and the temperature at both sides of the sensor. The temperature of each of these two copper plates is also recorded to correctly translate voltage measurements in the sensor to heat flux using the calibration files. The electrical power of the TPV cell is measured by a Keithley 2400 4-probe source-meter. A Peltier cooler controlled by PID and a water-cooled heat sink are used so the temperature of the TPV cell remains stable at appointed set point.

To test the experimental setup, we have employed an incandescent lamp (Osram Halospot $(111$ ) and a focusing lens (Fig. 1(b)) to emulate the high irradiances of an incandescent thermal emitter. A refractory ceramic mask over the TPV cell is used to ensure that the incident radiation is absorbed in the TPV cell only. Thus, all the heat flux measured by the sensor corresponds to the heat flowing through the TPV cell. The convection heat losses from the front TPV cell surface are minimized by introducing the setup inside a vacuum chamber $(\sim 0.1 \mathrm{mbar})$. The measurements have been conducted on commercial $\mathrm{GaSb}$ TPV cells of $\sim 1.5 \mathrm{~cm}^{2}$.

\section{RESULTS AND DISCUSSION}

With the objective of calibrating the setup, we have conducted a test consisting of direct-biasing the GaSb cell, in a total absence of light, and then measuring the variation of the heat flux. In the ideal case, we would work under the assumption that all the power supplied is dissipated by the cell and then is transmitted through the heat flux sensor. Fig. 2 shows the relationship between the heat flux in the system for both ambient and vacuum conditions. In both cases, the measured heat flux increases linearly with the electric power supplied to the TPV cell. In addition, and as expected, the heat flux is slightly greater when the system is subjected to vacuum conditions. This is due to a reduction of the convective thermal losses from the TPV cell front surface. However, not all the power supplied to the cell is registered as heat by the heat flux sensor. For vacuum conditions the heat flux amounts to $\sim 78 \%$ of the supplied power, while without vacuum it reaches $\sim 74 \%$. The reaming power losses ( $22 \%$ in vacuum) might be attributed to a variety of factors: i) the electro-luminescence of the cell (LED-like emission). ii) The resistive losses in the wires, which doesn't contribute to the measured heat flux. We expect this contribution to be small because we adopted a 4-probe I-V measurement configuration, which ensures that the measured electrical power corresponds to the power actually dissipated in the cell. iii) Heat conduction through the wires that contribute to dissipate part of the heat generated in the TPV cell. This contribution is also expected to be small, given that the cell is maintained at $25^{\circ} \mathrm{C}$, so that there is no large temperature gradient through the wires that could result in noticeable heat flows. And lastly iv) leakage heat losses through the other parts of the setup. Depending on the source of these heat losses, it would be necessary to implement a heat correction factor to obtain truthful heat flux measurement. In the case of the electro-luminescence, most of the emitted radiation would be absorbed by the thermal emitter, therefore it wouldn't entail energy losses for the system and no heat 
correction factor would be required. However, as we will prove further ahead, the energy losses due to the electroluminescence of the cell are negligible compared to the other sources of losses. Therefore, in order to correctly present the results for the TPV efficiency, a correction factor must be included that accounts for these heat losses. Therefore, $Q_{\text {corr }}=k Q_{\text {meas, }}$, being $k=1.28(k=1.35)$ for vacuum (non-vacuum) conditions, according to the results obtained from Fig. 2.

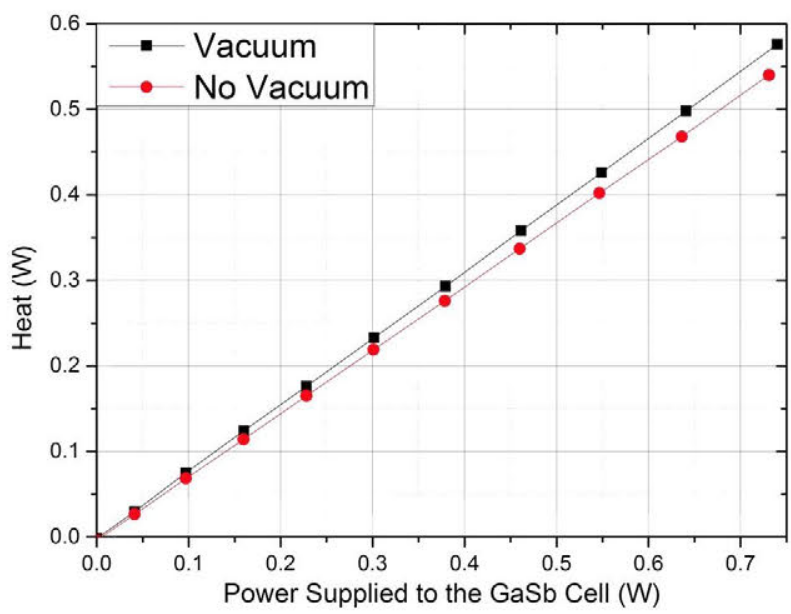

Fig. 2. Heat flux in the system vs power supplied to the GaSb cell in absence of light.

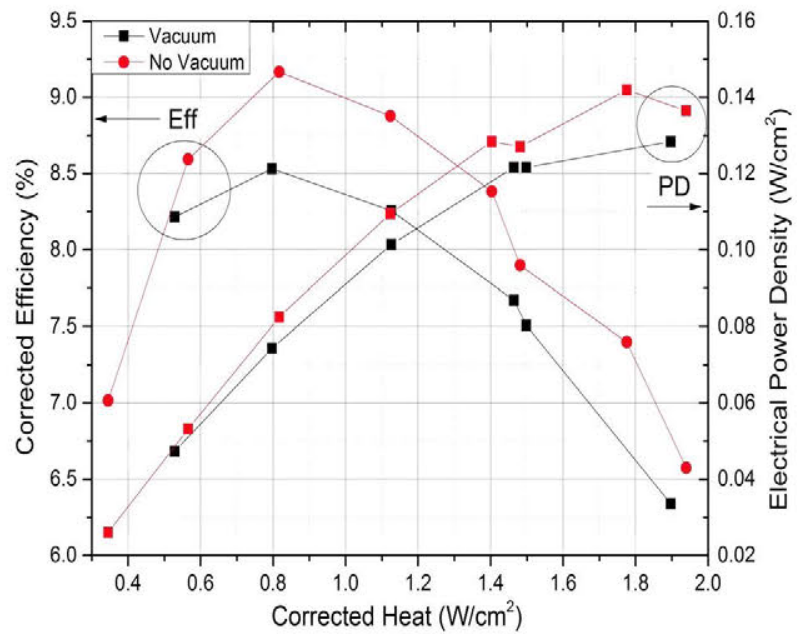

Fig. 3. Efficiency and electrical power density of the GaSb cells vs the heat flow in the system.

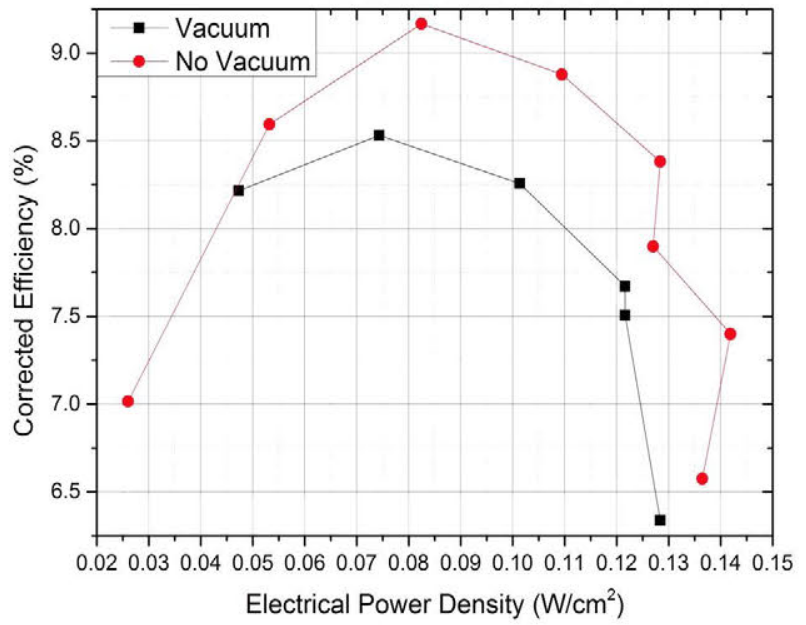

Fig. 4. Efficiency of the GaSb cells vs the electrical power density in the system.
Fig. 3 shows the (corrected) TPV conversion efficiency and the electrical power density at the maximum power point (MPP) for the TPV cell (maintained at $25^{\circ} \mathrm{C}$ ) versus the (corrected) heat flux $\left(Q_{\text {corr }}\right)$. Fig. 4 shows the conversion efficiency as a function of the electric power density. In these plots, each value of heat flux (or power density) corresponds to a different irradiance from the halogen lamp. The measurements were conducted in steady state and for a constant cell temperature of $25{ }^{\circ} \mathrm{C}$. Each point in these graphs required about 10 minutes to reach the steady state. It can be seen that the efficiency has a maximum at a certain value for the incident radiant power, which corresponds to a heat flux (electrical power density) of $\sim 0.82 \mathrm{~W} / \mathrm{cm}^{2}(\sim 0.074$ $\left.\mathrm{W} / \mathrm{cm}^{2}\right)$ without vacuum and $\sim 0.80 \mathrm{~W} / \mathrm{cm}^{2}\left(\sim 0.082 \mathrm{~W} / \mathrm{cm}^{2}\right)$ with vacuum. It is well known that the linear (logarithmic) dependence of the current (voltage) on the incident irradiance causes a supra-linear dependence of the output power versus the incident irradiance that ultimately results in an increase of the PV conversion efficiency. This increase takes place until series resistance effects become noticeable, which causes the conversion efficiency to decrease due to ohmic losses at high irradiances. These effects are present in the results of Figs. 3 and 4 . However, it must be noticed that the power of the lamp depends supra-linearly with the filament temperature $\left(\sim T^{4}\right)$. Thus, the incident spectrum changes significantly for different incident irradiances. Since GaSb TPV cells absorb only photons with wavelengths shorter than $\sim 1800 \mathrm{~nm}$, a higher portion of the incident spectrum is wasted (transformed into heat that is measured by the sensor) at lower incident irradiance and lamp filament temperatures. All these phenomena also impact on the results represented in Figs. 3 and 4.

Figs. 3 and 4 also show that the TPV conversion efficiency measured by the setup falls when the system is introduced in vacuum conditions. In ambient conditions, the heat flux in the setup is reduced due to the convective heat losses from the TPV cell front side. When these heat losses are eliminated, a higher amount of heat must de dissipated from the bottom of the TPV cell, and, therefore, the measured efficiency drops. This proves that vacuum provides a more accurate characterization of the TPV cell's efficiency.

This setup for the characterization of the TPV conversion efficiency is based in the idea of that all the net radiation that it is absorbed by the TPV cell turns in either electrical power output or heat flux that runs through the sensor. All the power reflected back would be absorbed and reemitted by

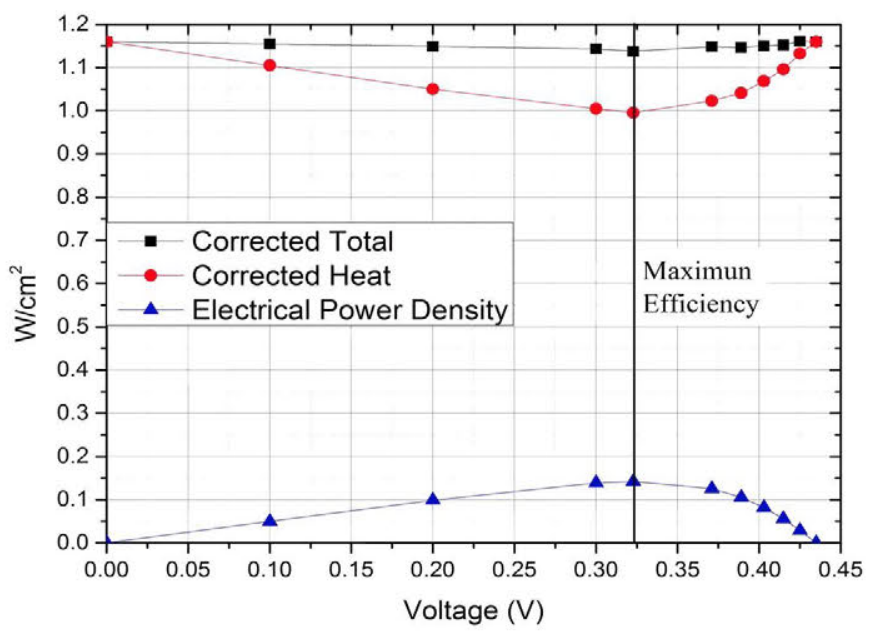

Fig. 5. Comparative Graph of the Heat Flow, Electrical Power and Total Power in the system. 
the thermal emitter. With the purpose of proving this concept, we have varied the voltage bias of the TPV cell and measured the variations on the generated electrical power and heat flux. Fig. 5 shows the generated electrical power, the (corrected) heat flux and the total power (heat plus electrical power) as a function of the TPV cell voltage bias. We observe how the heat flux diminishes as the voltage bias increase until it reaches a minimum and then increase again when we move forward to the open circuit voltage. The maximum power point possesses the lowest heat flux and the highest conversion efficiency, as expected. It is worth noticing that the value of the heat flux at both points where the generated electrical power is zero (open circuit and short circuit) coincides. This suggests that electro-luminescence is not very significant in this particular device. If it were, a significant decrease of the heat flux would be observed near open-circuit, corresponding to a significant rise of the power radiated by luminescence. This result justifies the use of the heat correction factor applied to the measurements discussed so far. Remember that electro-luminescent radiative power is not considered lost in a TPV device, because it contributes to reduce the net radiative flux between the emitter and the TPV cell.

We can also observe a slight decrease of the total power shown in Fig. 5 at the MPP, which is attributed to a slight underestimation of the electrical power and/or the heat flux. This small deviation might be explained by the specific location of the sense probes, which impact on the precise determination of the electrical power generated in the TPV cell. Also, some part of the Joule losses in the wires might not be dissipated through the sensor. This suggests that a polarization-dependent correction factor might be needed in future system designs.

\section{CONCLUSSION}

We have described a new experimental method to measure directly the TPV conversion efficiency. Despite having used an incandescent lamp instead a thermal emitter, preliminary experimental results using this method indicate great potential to achieve precise and reliable measures of TPV conversion efficiencies, which hadn't been available until now. We have confirmed that the TPV cell shows an expected behavior when is subjected to different irradiance from the halogen lamp, displaying a maximum conversion efficiency for a determined irradiance while the generated electrical power and the heat flux monotonously increase. The changes in performance when vacuum conditions are applied also coincides with the foreseen prospects of this work. Lastly, we have also asserted that the total power (heat plus electrical power) measured remains practically constant regardless of the working conditions (applied voltage bias) of the cell. Future work will consist of using this method to measure an actual TPV device, comprising a TPV emitter instead of a lamp as the photon source.

\section{ACKNOWLEDGMENT}

This work has been partially funded by the project AMADEUS, which has received funds from the European Union Horizon 2020 research and innovation program, FETOPEN action, under grant agreement 737054. The sole responsibility for the content of this publication lies with the authors. It does not necessarily reflect the opinion of the European Union. Neither the REA nor the European Commission are responsible for any use that may be made of the information contained therein. A. Datas acknowledges the current postdoctoral fellowship support from the Spanish "Juan de la Cierva - Incorporación" program (IJCI-201523747).

\section{REFERENCES}

[1] T. Bauer, "Thermophotovoltaics: Basic Principles and Critiical Aspects of System Design". Springer, 2011.

[2] D. L. Chubb, "Fundamentals of thermophotovoltaic energy conversion". Elsevier, 2007.

[3] Bernard Wernsman, "Greater Than 20\% Radiant Heat Conversion Efficiency of a Thermophotovoltaic Radiator/Module System Using Reflective Spectral Control", IEEE Transacrtions on Electron Devices, vol. 51, No. 3, 2004.

[4] L. M. Fraas, J. E. Avery, H. X. Huang, and R. V. Martinelli, "Thermophotovoltaic system configurations and spectral control," Semicond. Sci. Technol., vol. 18, pp. S165-S173, 2003.

[5] Z. Chen, P. L. Adiar, and M. F. Rose, "Multiple-dopant selective emitter," in Proc. NREL Thermophotovoltaic Gen. Electricity Conf.,vol. 401, AIP Conf. Proc., 1997, pp. 181-188.

[6] D. E. Pierce and G. Guazzoni, "High temperature optical properties of thermophotovoltaic emitter components," in Proc. NREL Thermophotovoltaic Gen. Electricity Conf., vol. 460, AIP Conf. Proc., 1999, pp. 177-190.

[7] R. R. Siergiej. B. Wernsman, S. A. Derry, R. G. Mahorter, R. J. Wehrer, S. D. Link, M. N. Palmisiano, R. L. Messham, S. Murray, C. S. Murray, F. Newman, J. Hills, and D. Taylor, " $20 \%$ efficient InGaAs/InPaS thermophotovoltaic cells", in Proc. Thermophotovoltaic Gen. Electricity Conf., vol. 653, AIP Conf. Proc., 2003, pp. 414-423.

[8] C. Ferrari, F. Melino, M. Bosi, "The critical role of emitter size in thermo-photovoltaic generators", Solar Energy Materials \& Solar Cells 113, 2013, 20-25

[9] R. G. Mahorter, B. Wernsman, "Thermophotovoltaic system testing", Bechtel Bettis, Inc., PO Box 79, West Mifflin, PA 15122, USA

[10] A. Datas, C. Algora, "Analytical Model of Solar Thermophotovoltaic Systems with Cylindrical Symmetry: Ray Tracing Approach", Prog. Photovolt: Res. Appl. 2009; 17:526-541 